\title{
Fat Finger Worries: How Older and Younger Users Physically Interact with PDAs
}

\author{
Katie A. Siek, Yvonne Rogers, and Kay H. Connelly \\ Indiana University, Bloomington, IN 47405, USA \\ \{ksiek, yrogers, connelly\}@indiana.edu
}

\begin{abstract}
There has been a steady growth in the global population of elderly people, challenging researchers in the HCI community to design technologies to help them remain independent and preserve their quality of life. One approach has been to create assistive technology solutions using Personal Digital Assistants (PDAs). However, some have questioned whether older people can use PDAs because of age related problems with dexterity, coordination, and vision. This paper presents an initial usability study that shows there are no major differences in performance between older and younger users when physically interacting with PDAs and completing conventional (e.g. pressing buttons, viewing icons, recording messages) and non-conventional tasks (e.g. scanning bar codes).
\end{abstract}

\section{Introduction}

Each month, the world's elderly population grows by 795,000 . By the year 2030 , the world's older population will grow by 847,000 per month [1]. Researchers in the HCI community have taken notice of this trend and are working on applications to help older people live independent and productive lives. Personal Digital Assistants (PDAs) [2,3] and smart phones [4] are some of the devices researchers use to create assistive technologies for older people. Our research is concerned with how PDAs can be used as personal aids for health informatics, in particular, for helping older people who have end-stage renal disease (ESRD) monitor their nutrition more effectively.

When we started our nutrition monitoring project, we were cautioned that older people may not be able to use PDAs given the adverse effects age can have on vision, dexterity, and coordination [5, 6]. If elderly populations have difficulties using traditional personal computers (PCs), as has been found in some studies, how will they fare when interacting with the smaller screen and buttons of a PDA? The lack of literature available on how elderly physically interact with PDAs led us to conduct an initial study to see if there were any differences between older and younger people when physically interacting with PDAs.

In this paper, we present the findings from our study investigating whether elderly people (75-85 years old) have problems using PDAs. As a control to compare older people with, a group of younger people (aged 25-30 years old) participated in the study. Participants were asked to complete three conventional PDA tasks (e.g. pushing buttons, viewing icons, and recording voice messages) and two additional tasks (e.g. scanning bar codes with two kinds of scanners). The scanning tasks were included in the study to 
(1) determine how easy it is to input nutrition information that is found on food items and (2) give insight into how older people would perform on other less familiar PDA tasks, such as taking digital pictures with the device.

The findings from our initial study suggest that older people completed the tasks nearly as well as younger participants. While older people needed more practice before completing each task, their performance was similar to the younger participants.

We begin with a review of related work. The technology, applications, and evaluation techniques we used in the study are discussed in Section 3. In Section 4, we discuss the user study and evaluate the results. We conclude with a discussion of the results and ideas for future work.

\section{Related Work}

There has been a proliferation of handheld devices designed for the general public, including PDAs, cell phones, remote controls, digital cameras, digital music players, and game playing devices. The interfaces to these vary considerably, suggesting there may be variable age-related performance effects. Hence, when creating applications for older populations that run on these devices, there is a need to consider age-related abilities such as vision, dexterity, coordination, and cognition. HCI researchers have acknowledged that within older populations, there are noticeable differences in abilities and that different design methodologies such as Universal Design [7] and User Sensitive Inclusive Design [8] should be used. Here we discuss some of the research that has been done to better understand older populations interaction with technology.

Bernard et al. found that older people could read faster with a larger, more legible 14-point san serif font on web sites [9]. Researchers at Georgia Tech studied how multimodal feedback (sound, touch, visual effect) could assist participants with varying vision problems perform basic mouse tasks (drag and drop). They found that all groups performed better when sound was added, however groups performed the best when all three modal feedbacks were implemented [10].

A number of recent studies [11, 12,13, 14] focused on the ability of older populations to use PC input devices. The studies showed that older people completed tasks more slowly when compared to younger groups. Charness et al. evaluated control key, mouse, and light pen input devices and found older people preferred the light pen followed by the mouse and control keys [13].

Smith et al. and Laursen et al. found older people made more mistakes than younger people and had the most difficulty with fine motor control tasks such as double clicking. However, Chaparro et al. found older people performed "point and click" and "click and drag" tasks slower than younger people, but with the same amount of accuracy. The researchers deduced that older people were slower because of the reduced fine motor control, muscle strength, and pincher strength associated with older age.

Most of the human computer interaction studies on elderly and technology have focused on the usability of PCs. As pervasive computing technology applications become more widespread, the usability of handheld devices will be scrutinized more carefully. Researchers are already assessing the needs of older people with respect to mobile phones. Maguire and Osman found that older people primarily considered mobile 
phones as a way to assist in emergencies, whereas younger people saw mobile phones as a way to interact socially. Older people were interested in small phones with large buttons and location aware systems [15].

Abascal and Civit looked at the pros (safety, increased autonomy, etc.) and cons (social isolation, loss of privacy, etc.) of older people using mobile phone technologies and gave a needs assessment [7]. The pros and cons apply to assistive PDA solutions as well, however the needs assessments differ because PDAs have larger physical interfaces and different input mechanisms.

Smith et al. and Maguire and Osman suggested voice input could assist with difficulties older users have with mouse and mobile phone input. Using PDAs for voice recordings is becoming a popular way to get user feedback in situ. For example, Intille et al. integrated voice recordings into their context-aware experience sampling tool for PDAs to obtain feedback from participants [16]. The natural decrease in pincher strength [17] and difficulty maintaining constant force [18] that accompanies old age may hinder older populations from using voice input technologies.

The findings from these studies suggest that there may be performance differences for older people when using small handheld devices. They may find it hard to manipulate small buttons that are close to one another and read small icons on a screen. PDAS have been designed to allow users to select from two size icons and input information using other kinds of devices besides keypads, keyboards, and mice (e.g. bar code scanners, touch screen). The aim of our study was to assess PDA input mechanisms that do not involved mouse movement or the cognitive mapping between mouse pad coordinates and screen position. PDAs also have the advantage of being all-in-one devices users can input commands and view output on the same devices. We also wanted to see if older people could successfully record voice messages using a PDA.

In particular, the goal of our usability study was to see whether vision, dexterity, and coordination effects transfer to PDAs or whether the ergonomic design of PDAs is adequately "large" enough to enable older populations who may have more dexterity, coordination, and vision problems. The specific aims were to:

- Compare performance between older and younger participants performing conventional (pushing buttons, viewing icons, and recording voice messages) and nonconventional (scanning bar codes with two different types of scanners) tasks

- Study how older and younger participants physically interact with PDAs (e.g. how they hold the device, how far away they hold the device, etc.) to understand any difficulties participants may have completing PDA tasks

\section{Experiment Design}

In this section, we discuss why we selected the Tungsten T3 PDA, Socket SDIO scanner and Baracoda pencil as our scanners, and how we developed the usability tasks. Detailed information about the experimental design can be found in our full report [19].

\subsection{Hardware}

PDA Selection PDAs are the center of our study and the building block of many pervasive computing applications. Most HCI PDA applications use off-the-shelf PDAs to 
make their application more widely accessible and less expensive. The physical design of PDAs are similar because they have small screens with five buttons. The middle button is usually larger than the other four and (in newer models) is a 5-way navigator buttons. The 5-way navigator buttons allow users to scroll through applications and documents with one hand. We conducted the study with an off-the-shelf PDA so the results would be useful to the HCI community. We chose the Tungsten T3 because it has a 5-way navigator button, four large application buttons, Bluetooth, an SDIO slot, and a voice recorder.
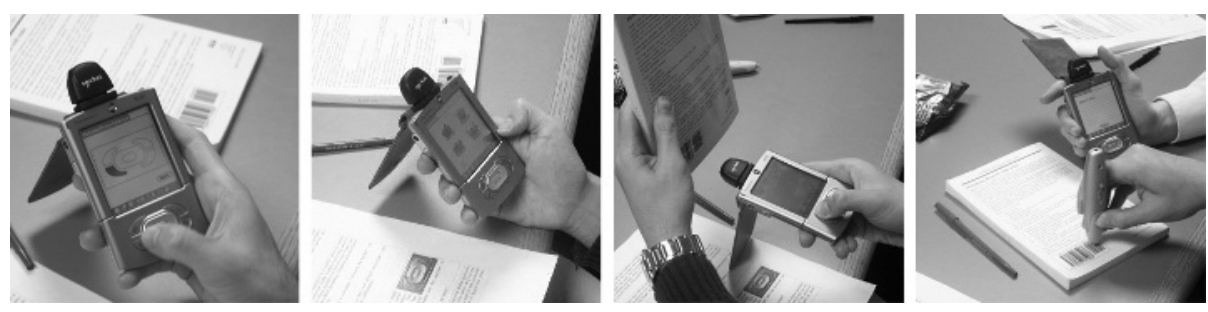

Fig. 1. Usability test tasks: (L-R) button press, icon size, Socket scanning, and Baracoda scanning

Scanner Selection A scanner needs to be small, easy to use, and robust for integration into HCI applications. We found two scanners that met our criteria - Socket SDIO card scanner and the Baracoda pencil. To operate the Socket scanner, users press the predefined PDA scanning button and line up the scanning light with the bar code as shown in Figure 1. The PDA beeps and shows the bar code number on the screen when users have successfully scanned the bar code. To operate the Baracoda pencil scanner, users press a button on the side of the Baracoda pencil and run the pencil tip over the bar code as shown in Figure 1 The users must look at the PDA screen to see if they have successfully scanned the bar code.

\subsection{Designing the Applications}

People must have a basic level of dexterity, coordination, and vision to use a PDA. We tested these three aspects by asking people to complete a set of tasks summarized in Table 1. The tasks were designed to measure primarily motor control, rather than

Table 1. Types of tasks and the characteristics needed to successfully complete the task

\begin{tabular}{l|l} 
Type of Task & Ability Needed To Complete Task \\
\hline Push PDA buttons & Dexterity \\
Selecting an icon & Vision \\
Recording a voice diary entry & Dexterity \& Coordination \\
Socket Scanning bar code & Dexterity \& Coordination \\
Pencil Scanning bar code & Dexterity \& Coordination
\end{tabular}


mental effort. We chose not to investigate stylus input because we considered it might be too intimidating for first-time users (e.g. learning graffiti). In this section, we discuss how we designed and evaluated the five different input tasks.

PDA Button Press Task. We tested whether participants could press buttons on the PDA because buttons are the primary input method for accessing applications and scrolling through data. We developed an event-driven test modeled after the 1980's Simon Says game, shown in Figure 2, to test the ability to press buttons. The picture on the PDA screen showed the same configuration of buttons as the buttons on the Tungsten T3. The buttons take turns "lighting up" by turning red until the participant selects the corresponding button on the PDA. The task tests if the participant can press each of the nine Tungsten T3 buttons once (four buttons and each of the 5-way navigator buttons). Errors (e.g. if a participant pushes the incorrect button) were recorded as shown on the last screen image in Figure 2 We also recorded how far away the participant held the PDA and what hands and or fingers the participant used to complete the task. We examined how participants held the PDA to see how comfortable the participants were interacting with the device.

Icon Size Task. Icons are used to select PDA applications from the main menu and navigate within applications. Moreover, icons can convey information to users independent of literacy skills. We created a task similar to an eye exam chart, shown in Figure 3 , to test what size icons participants prefer to use (vision). Icon sizes ranged from $5 \mathrm{~mm}$ to $25 \mathrm{~mm}$. Current PDA icons are $7.76 \mathrm{~mm}$ or $5.29 \mathrm{~mm}$ square depending on the layout

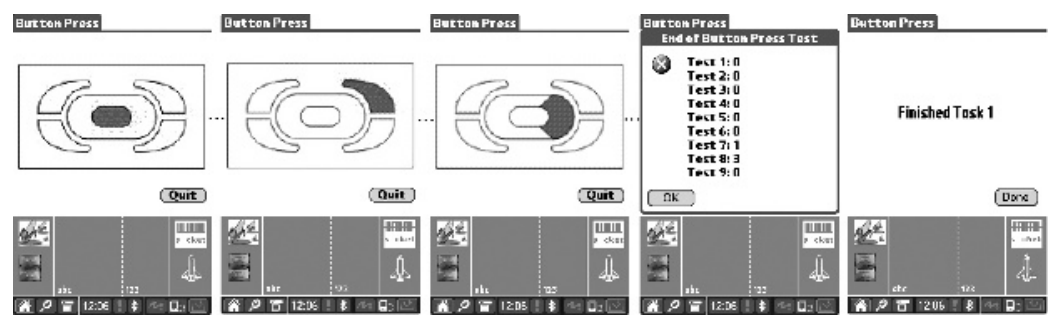

Fig. 2. Button Press Task. The last screen indicates the errors that were made.

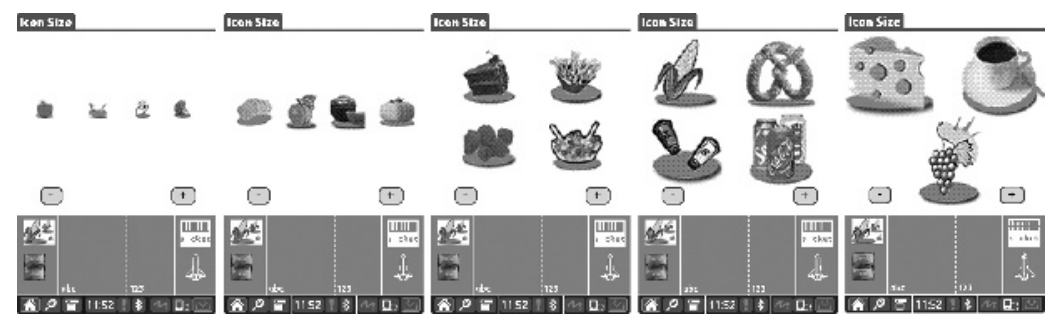

Fig. 3. Icon Size Task. From left to right: $5 \mathrm{~mm}, 10 \mathrm{~mm}, 15 \mathrm{~mm}, 20 \mathrm{~mm}$, and $25 \mathrm{~mm}$ screens. 
chosen [20]. When the task was started, a screen with four $15 \mathrm{~mm}$ icons was displayed. The participant was asked to read the pictures on the screen. We increased or decreased the size of the icons based on the participants answers. The task concluded by recording what size icons the participants preferred, the smallest icon size the participants could read, how far the participants held the PDA from them, and if they had a preference between the realistic pictures or the illustrated drawing icons. We noted preferred icon size and what sizes the participants could read to see if there were any noticeable differences between their preference and vision.

Recording a Voice Diary. For this task, we asked participants to record comments and questions to see how the participants created voice diaries. To do this requires pressing the record button on the side of the PDA, waiting for a beep, and then continuing to hold the button down while recording a voice diary. We asked participants to record three phrases - a short phrase (approximately 1 second), a medium length phrase (approximately 5 seconds), and a longer phrase (approximately 15-20 seconds). After recording each phrase, the participants were asked to play it back. If the participants did not record the message properly, they could try recording the phrase again. We recorded how many times participants successfully recorded each phrase, if the participants waited for the beep before saying each phrase (learning), how far the participants held the PDA away from them, and any difficulties the participants had finding the recording button.

Scanning Items. Scanning bar codes is an easy input mode that does not require intensive cognitive effort to choose items from a menu. However, participants must have a reasonable level of manual dexterity and coordination to scan an object because participants must hold the scanner steady (dexterity) and work with two objects - the scanner and object being scanned (coordination). For the usability test, participants were asked to scan three items: a book, a small bag of pretzels, and a can of soda. Participants had to hold each item differently because the properties varied among items (e.g. one was hard, another mushy, and the other curvy and reflective). We were interested in seeing how the physical properties of the object affected their ability to scan the items. We recorded how many times it took participants to successfully scan each item, if the participants moved the PDA or object being scanned, and how many times they practiced scanning an item after successfully scanning an item the first time.

\section{PDA Usability Study}

The study required the participants to complete a set of tasks testing their ability to use PDA buttons, view icons, record a message, and scan items with two types of scanners. Younger participants were tested in a meeting room in an academic building. Older participants were tested in a meeting room in an assisted living community building.

\subsection{Hypotheses}

Based on the literature that showed elderly people can use PCs equally, we hypothesized that there would be no difference between the two age groups. We predicted that: 
- Participants of all ages can press buttons on the PDA.

- Participants of all ages prefer medium size $(10 \mathrm{~mm}$ or $15 \mathrm{~mm})$ icons.

- Participants can record voice messages of various lengths.

- Participants can scan bar codes with some practice.

\subsection{Participants}

Twenty participants volunteered for the study. The control group consisted of ten participants 25-30 years old (two female, eight male). The older group had ten participants 75-85 years old (five female, five male). We chose a younger group to compare them with as they have normal dexterity, vision and coordination and therefore, would be able to use PDAs with ease.

Sixteen participants reported using computers a lot. Younger participants used computers primarily for word processing, emailing, and Internet searches. Older participants used computers primarily for emailing and viewing images. We asked how often and what kind of applications participants used to get a more accurate measure on computer experience (scaled 0-3: 0 - not at all; 1 - not often with 1 application; 2 - sometimes with 1-2 applications; and 3 - often with various applications). We found participants had similar computer experience $\left(\mathrm{T}_{18}=1.24, \mathrm{p}=0.232\right)$.

None of the participants in the two groups owned a PDA. However, three younger participants reported they had some experience with PDAs (e.g. occasionally played with a friend's PDA by playing a game or drawing a picture).

All of the older participants and over half of the younger participants wore glasses when using a computer, but did not report any problems reading computer screens. None of the participants had problems using television remote controls. Over three quarters of the younger participants and three of the older participants used cell phones without any difficulty.

\subsection{Design and Procedure}

Participants completed the five tasks without any time constraints. We did not enforce any maximum amount of viewing time because we wanted the participants to feel comfortable reading the icons and avoid the stress associated with timed events. Laursen et al. found placing time constraints on older people increase the number of errors [14]. Each participant was given a task sheet describing what to do for each task. We let participants hold the PDA for each task as shown in Figure 1

Since both groups had experience pushing small buttons on cell phones and remote controls, we allowed participants only one chance to complete the button press task. In addition, the button press task gave us insight into how intuitive it was for participants to hold the PDA.

For the icon size task, participants held the PDA and moved the PDA to see the icons clearly. The task administrator or participant changed the size of the icons by pressing PDA buttons.

During the voice diary task, participants read aloud three phrases printed on the task sheet. Participants played back their recording to ensure they successfully recorded the messages. Participants recorded each phrase as many times as they wanted. 
During the scanning tasks, participants scanned the bar codes on three items: a book, a small bag of pretzels, and a can of soda. Participants scanned each object as many times as they wanted so they could practice and become familiar with the scanning device. They were encouraged to start the task by scanning the book, then the bag of pretzels, and finally the soda can because each item was increasingly difficult to scan based on bar code material and object size.

At the end of the tasks, we discussed the comments the participants made during the study.

\subsection{Findings}

As predicted, the key findings from our study were that:

- There were no differences in performance between the older and younger groups for the button press and voice recording task.

- We also found some small differences between the two groups: The younger participants preferred smaller icons $(5 \mathrm{~mm}$ or $10 \mathrm{~mm})$, whereas the older participants preferred larger icons $(20 \mathrm{~mm})$.

- The older participants scanned items more with both scanners, but had the same success rate as younger participants.

In this section, we look in more detail at the results for each task. The results are presented in the order the tasks were completed - button press, icon size, voice recording, Socket SDIO scanning, and Baracoda pencil scanning.

Button Press Task. The button press results supported our hypothesis by showing no significant performance differences between older and younger participants - 8 participants from each group did not make any mistakes during the task. All participants voiced some confusion over the 5-way navigator button. The button press task started by making participants press the middle of the 5-way navigator button, thus participants knew the 5-way navigator button was different than the other buttons. When participants saw the up or down part of the 5-way navigator light up they made comments about how the navigator is an "up and down" button. However, when the left and right part of the 5-way navigator lit up, participants voiced some concerns. Most participants followed their instincts and pressed the left or right part of the large navigation button, but three participants pressed the incorrect button. The participants who pressed the incorrect button learned quickly from their mistakes.

The older male participants voiced concerns about how their "fat fingers" may cause problems when completing the task. They worried that the size of their fingers would cause them to push multiple buttons at the same time. However, the "fat finger" problem was not supported since not many errors were recorded during the task. As previously stated, the only errors were caused by the 5-way navigator button.

Most of the participants held the PDA in their non-dominant hand and selected buttons with their dominant hand. Only three younger participants completed the task by using one hand to hold the PDA and select buttons. This was the first task participants completed with the PDA, thus they were not as comfortable with holding the PDA. 
A t-test indicated that there were no significant performance differences in terms of incorrect button presses due to age $\left(T_{18}=0.787, \mathrm{p}=0.442\right)$. Participants commented that the task was "easy to follow" - the PDA told them exactly what to press and 9 of the older participants said the button press task was the easiest task they completed during the study.

Icon Size Task. Results from the icon size task were a little surprising. Our hypothesis was confirmed by younger participants who preferred icons $10 \mathrm{~mm}$ (mean $=10 \mathrm{~mm}$, standard deviation $=3.33 \mathrm{~mm}$ ). However, older participants preferred icons $25 \mathrm{~mm}$ (mean $=18.5 \mathrm{~mm}$, standard deviation $=6.687 \mathrm{~mm}$ ). Despite the older participants preferring larger icons, they were all able to read icons $\leq 15 \mathrm{~mm}$ (younger: mean $=5.5 \mathrm{~mm}$, standard deviation $=1.581 \mathrm{~mm}$; older: mean $=10 \mathrm{~mm}$, standard deviation $=4.082 \mathrm{~mm})$. A ttest indicated that there were significant differences in icon size preference $\left(T_{18}=3.73\right.$, $\mathrm{p}=0.002)$ and the size icon they could actually see $\left(T_{18}=3.25, \mathrm{p}=0.004\right)$.

When we asked participants why they chose a specific size icon, the younger participants were interested in how many icons could fit on the screen. Older participants were primarily interested in larger icons so they could "clearly see details." This accounts for the preferences of size.

The icon size application used scaled photographs and illustrations of food items as shown in Figure 3 to see if participants had a preference. A majority of the younger group did not have a preference, but the older group preferred the photographs because they were "clearer" and "more realistic."

During the icon size task participants were allowed to hold the PDA to view the icons. Both groups of participants held the PDA at about the same distance on average (younger: mean $=14.8$ ", standard deviation $=6.339$ "; older: mean $=12.5$ ", standard deviation $=4.249$ "). The only noticeable difference in how the participants held the PDA was the older group tilted the PDA in their hand trying to view the icons with less glare. The younger group did not have a problem with glare.

Recording a Voice Diary Entry Task. Participants were asked to record three phrases during the recording voice diary entry task - a short phrase, a medium length phrase, and a longer length phrase. The voice diary recording task was an easy task for most of the participants - 7 participants from each group were able to record the short message correctly the first time. Participants who could not successfully record the short message during their first try, succeeded on their second try. All of the younger participants and all but one of the older participants successfully recorded the long messages on their first try. Overall, we found no significant performance differences in successfully recording all three messages $\left(\mathrm{T}_{18} \leq 0.5, \mathrm{p}>0.3\right.$ for all three recordings).

Most of the younger participants held the PDA in their left hand and used their thumb to press the button when recording the messages. A majority of the older participants used two hands when recording - the right hand stabilized the PDA while the left hand pressed the recording button. Some older participants expressed a fear of breaking the PDA and held it with two hands to make sure they had a good grip on the device. The fear of breaking the PDA could attribute to why most people in the older group used two hands for some of the tasks. Both groups held the PDA about nine inches away on average from themselves when recording their messages. 
Socket SDIO Scanning Task. The older group was more successful in scanning the book on the first try than the younger group (50\% versus $40 \%$ success rate). Nine younger and 10 older participants were able to scan the book in less than four attempts. Younger participants were able to scan the bag of pretzels on the first try better than older participants (60\% versus $50 \%$ success rate). All of the participants were able to scan the the bag of pretzels eventually. The can of soda was the most difficult item to scan - only 4 of the younger participants and 2 of the older participants were able to scan the can within three tries. Overall, we found no significant performance differences in all three scannings (book: $T_{18}=0.958, \mathrm{p}=0.351$; bag: $T_{18}=0.247, \mathrm{p}=0.808$; can: $\left.T_{11}=1.30, \mathrm{p}=0.221\right)$.

We observed how practice affects scanning success when we compared the number of successful book scans participants completed before trying to scan the bag of pretzels. Younger participants who practiced successfully scanning the book 3-8 times were able to scan the bag of pretzels more quickly (successfully scanned bag after 1.6 attempts) than those who only practiced successfully scanning the book 1-2 times (successfully scanned bag after 2.6 attempts). Half the younger and older participants practiced scanning the book three or more times before trying to scan the bag. We did not find any relation between overall scanning practice and the ability to successfully scan the soda can. The soda can was an especially challenging item to scan because of its curved edges and reflective material.

Most participants operated the scanner with one hand, using their thumb to press the scanning button on the PDA. The older participants liked the multimodal feedback the scanner provided. They used the scanner light to indicate the distance needed for a successful scan and the beep as a way of ensuring they were successful scanning the bar code. All of the participants exhibited some confusion on what part of the bar code to scan (numbers or lines) and what direction to scan the bar code (sweeping vertically or shine the scanner light across the bar code). When scanning items, older people kept the scanner still and moved the item being scanned, whereas the younger people moved scanner and kept the item stationary. Even though the older participants completed $66 \%$ more scans (successful and unsuccessful) than the younger group, they were not frustrated by the activity and felt they did the best they could.

Baracoda Pencil Scanning Task. The Baracoda pencil scanner task was the most difficult task. Only two younger participants and one older participant successfully scanned the book on the first attempt. On average, younger participants attempted to scan the book more than older participants (4.2 times versus 3.3 times) before successfully scanning the bar code on the book. A t-test indicated that there were no significant performance differences due to age when scanning the book $\left(T_{17}=0.430, \mathrm{p}=0.672\right)$.

Additionally, older participants attempted to scan the bag of pretzels more than the younger participants before giving up (15.4 times versus 8.8 times). Only three participants were able to successfully scan the bag of pretzels. Participants inability to scan the bag of pretzels and soda can simply shows the limitations of the device.

Two female participants had difficulty scanning with the Baracoda pencil because the length of their nails (the length of nails ranged from an $\frac{1}{8}$ " to $\frac{1}{4}$ ") inhibited them from depressing the narrow button fully. Female participants held the pencil precariously and used the tip of their nail to press the button. Older participants usually pressed the 
scanner button, reflected the scanner light on their hand, and then scanned the item. They commented that they wish they could see the scanner light while scanning.

Scanning with the Baracoda pencil scanner required the use of a pencil scanner and the PDA. The PDA did not give any audible feedback on successful scans, thus participants had to check the PDA screen to see if they had successfully scanned the object. Participants rested the PDA and item on the table when scanning. From our observations, it appears that the Baracoda pencil scanner would be difficult to use while standing or moving.

\section{Discussion}

The results of our preliminary study supported our null hypotheses. Concerns that older participants would have difficulty pressing the PDA buttons because of decreases in dexterity with age or the similarity of the layout between PDA buttons and PC control keys [13] were unfounded. Similar to the study by Chaparro et al., we found that older participants were able to select the correct button (fine motor control) and push the button while holding the PDA (pincher strength) with the same accuracy as younger participants [12]. We also found no difference between age groups: both older and younger participants performed at the same level in the button press task.

Participants preference for icon size was the only hypothesis that was rejected younger participants preferred smaller icons $(5 \mathrm{~mm}$ or $10 \mathrm{~mm})$ and older participants preferred larger icons $(20 \mathrm{~mm})$. The older participants preferred larger icons in comparison with the younger participants because it was easier to see the details. Younger people are also more familiar with distinguishing small graphical images from various applications they use. Our findings are similar to those reported by Czaja and Lee who looked at numerous PC studies on vision and the elderly [21]. During the icon size task, we noted that older people tilted the PDA to view the icons with less glare. Older people's sensitivity to glare was also recorded in a study by Kosnik et al. [5]. The icon size task showed us a one-size-fits all approach to developing PDA applications does not work. Current PDA applications offer two different size icons, but both of these sizes are smaller than the preferences of our two groups.

Similar to the button press results, assumptions that older participants may not do as well recording a message because of age related difficulty maintaining a constant pincher force [18] were not evident in our study. Older and younger participants were able to record voice messages with similar performance rates. The difference in how the two groups held the PDA (two hands for older participants and one hand for younger participants) could be attributed to grip strength. Mathiowetz et al. found high correlations between grip strength in age and observed that younger people had over $50 \%$ more grip strength than older people in our age groups [17]. Our participants showed that they could hold down the recording button (dexterity) while interacting with the PDA (coordination).

All of the participants were capable of scanning bar codes with some practice using the Socket SDIO scanner with no major differences in performance. Participants showed that they could hold the PDA steady (dexterity) while interacting with the PDA and object being scanned (coordination). Older participants liked the multimodal feedback (sound, visual effect) the PDA made when participants successfully scanned a bar 
code. Jacko et al. found that people in various age groups performed PC input tasks better with multimodal feedback (sound, touch, visual effect) [10]. Similar to PCs, PDAs have the ability to emit sound, vibrations, and visual feedback to assist participants perform better.

When we selected scanners for the study, we based our selection on product documentation and reviews. Our study showed the Baracoda pencil's scanning (inability to scan a bag of pretzels and can of soda efficiently) and usability (small button made it difficult to scan with longer nails) limitations. The Baracoda pencil scanner was equally hard for both participants. In future studies we will discontinue the use of the Baracoda pencil and add other tasks such as standing or walking when completing tasks.

This was the first time the participants had used a PDA for an extended period of time. We surmise with more practice, the participants would be able to easily scan all three items. Application developers can learn from our study: (1) older people can complete conventional and non-conventional PDA tasks and (2) applications for a wide range of participants need more icon sizes to select from to ensure universal usability.

The study investigated whether elderly people can physically interact with PDAs as well as younger people. Gick et al. found that the performance of younger and older people are similar when cognitive tasks are not complex [22]. We designed the tasks to emphasize motor control, not mental effort. Thus, we conclude that the participants are performing at similar levels because older participants can physically interact with PDAs at the same level as young novice PDA participants.

\section{Future Work}

In the future we would like to test more participants and investigate cognitive oriented tasks. We would also like to recruit 25-30 year old people from outside of the university and 75-85 year old people who live on their own or live in a public assisted living facilities instead of private assisted living facilities to diversify the participant pool.

Other avenues of research could include having participants stand and walk during tasks. Testing participants while standing and walking may affect icon size preference [23] and the ability to find and push correct buttons for the button press and voice recording applications. Scanning bar codes may also become more difficult when standing because participants will not have anything to balance their arm or the item on when scanning the bar code. Scanning bar codes while standing is important for us to evaluate because participants using our nutritional monitoring application must be able to input data anywhere - standing while preparing food, buying a can of soda from a machine, etc.

\section{Conclusion}

Researchers in the HCI community question whether older people can use PDAs given that they can have reduced vision, dexterity, and coordination. Our findings showed this not to be true: older participants can physically interact with PDAs the same as younger participants, with no major impediments. Our results can be used as a guidelines for creating applications for diverse age groups. 


\section{Acknowledgments}

We would like to thank the participants from Bell Trace Senior Living Community, Meadowood Retirement Community, and Indiana University. Katie Siek is supported in part by a NPSC fellowship and Sandia National Laboratories/CA. This work was supported by NSF grant EIA-0202048 and by a grant from the Lilly Endowment. We would like to thank Dorrie Hutchinson, Yu-Hsiu Li, and Kelli Gehlhausen for their help during usability testing. The authors would like to thank Jeremy Siek and the referees for their careful reading of this paper and for their well-considered comments.

\section{References}

1. Kinsella, K., Velkoff, V.A.: An aging world: 2001. Technical report, U.S. Dept. of Health and Human Services, National Institutes of Health, National Institute on Aging, U.S. Dept. of Commerce, Economics and Statistics Administration, and U.S. Census Bureau (2001)

2. Carmien, S., Gorman, A.: Creating distributed support systems to enhance the quality of life for people with cognitive disabilities. In: UbiHealth 2003. (2003)

3. Coroama, V., Rothenbacher, F.: The chatty environment - providing everyday independence to the visually impaired. In: UbiHealth 2003. (2003)

4. Helal, S., Giraldo, C., Kaddoura, Y., Lee, C.: Smart phone based cognitive assistant. In: UbiHealth 2003. (2003)

5. Czaja, S.J.: Computer technology and the older adult. In Helander, M., Landauer, T., Prabhu, P., eds.: Handbook of Human-Computer Interaction. 2nd edn. Elsevier Science, B.V. (1997) 797-812

6. Faye, E.E., Stappenbeck, W.: Normal changes in the aging eye. http://www.lighthouse.org/aging_eye_normal.htm (2000)

7. Abascal, J., Civit, A.: Universal access to mobile telephony as a way to enhance the autonomy of elderly people. In: Proceedings of the 2001 EC/NSF Workshop on Universal Accessibility of Ubiquitous Computing: Providing for the Elderly. (2001)

8. Newell, A., Gregor, P.: Accessibility and interfaces for older people - a unique, but many faceted problem. In: EC/NSF Workshop on Universal Accessibility of Ubiquitous Computing: Providing for the Elderly. (2001)

9. Bernard, M., Liao, C., Mills, M.: The effects of font type and size on the legibility and reading time of online text by older adults. In: CHI 2001. (2001)

10. Jacko, J., Scott, I., Sainfort, F., Barnard, L., Edwards, P., Emery, V., Kongnakorn, T., Moloney, K., Zorich, B.: Older adults and visual impairment: What do exposure times and accuracy tell us about performance gains associated with multimodal feedback? In: CHI 2003. (2003)

11. Smith, M., Sharit, J., Czaja, S.: Age, motor control, and the performance of computer mouse tasks. Human Factors 41 (1999) 389-396

12. Chaparro, A., Bohan, M., Fernandez, J., Choi, S.: The impact of age on computer input device - psychophysical and psychological measures. International Journal of Industrial Ergonomics 24 (1999) 503-513

13. Charness, N., Bosman, E., Elliott, R.: Senior-friendly input devices: Is the pen mightier than the mouse? In: 103rd Annual Convention of the American Psychological Association Meeting, New York (1995)

14. Laursen, B., Jensen, B., Ratkevicius, A.: Performance and muscle activity during computer mouse tasks in young and elderly adults. European Journal of Applied Physiology 25 (2001) $167-183$ 
15. Maguire, M., Osman, Z.: Designing for older inexperienced mobile phone users. In Stephanidis, C., ed.: Proceedings of HCI International 2003, Mahwah, New Jersey, Lawrence Erlbaum Associates (2003) 22-27

16. Intille, S.S., Tapia, E.M., Rondoni, J., Beaudin, J., Kukla, C., Agarwal, S., Bao, L., Larson, K.: Tools for studying behavior and technology in natural settings. In Dey, A.K., Schmidt, A., McCarthy, J.F., eds.: UbiComp 2003: Ubiquitous Computing, Springer (2003) 157-174

17. Mathiowetz, V., Kashman, N., Volland, G., Weber, K., Dowe, M., Rogers, S.: Grip and pinch strength: Normative data for adults. Arch Phys med Rehabil 66 (1985) 69-72

18. Galganski, M., Fuglevand, A., Enoka, R.: Reduced control of motor output in a human hand muscle of elderly subjects during submaximal contractions. Journal of Neurophysicology 69 (1993) 2108-2115

19. Moor, K.A., Connelly, K.H., Rogers, Y.: A comparative study of elderly, younger, and chronically ill novice pda users. Technical Report TR 595, Indiana University (2004)

20. Rhodes, N., McKeehan, J.: Palm OS Programming. 2nd edn. O'Reilly (2002)

21. Czaja, S.J., Lee, C.C.: 21: Designing Computer Systems for Older Adults. In: The Human Computer Interaction Handbook: Fundamentals, Evolving Technologies, and Emerging Applications. Lawrence Erlbaum Associates, Mahwah, NJ (2003) 413-425

22. Gick, M., F.I.M. Craik, R.M.: Task complexity and age differences in working memory. Memory and Cognition 16 (1988) 353-361

23. Hall, A., Cunningham, J., Roache, R., Cox, J.: Factors affecting performance using touchentry systems: tactual recognition fields and systems accuracy. Journal of Applied Psychology 73 (1988) 711-720 